\title{
Guillain Barré Syndrome in a multiple myeloma patient after the first course of bortezomib therapy: A case report
}

\author{
XINGBIN DAI $^{1,2}$, XUEMEI SUN $^{1}$, HAIWEN NI $^{1}$ and XUEJUN ZHU ${ }^{1}$ \\ ${ }^{1}$ Department of Hematology, Jiangsu Province Hospital Affiliated to Nanjing University of Traditional Chinese Medicine; \\ ${ }^{2}$ Department of Internal Medicine, First School of Clinical Medicine, Nanjing University of Traditional Chinese Medicine, \\ Nanjing, Jiangsu, P.R. China
}

Received September 23, 2014; Accepted May 20, 2015

DOI: $10.3892 / 01.2015 .3634$

\begin{abstract}
Bortezomib is approved for treating relapsed multiple myeloma (MM) and mantle cell lymphoma, and clinical evidence has shown complete clinical responses in MM patients. Peripheral neuropathy is one of the most common adverse effects of bortezomib. However, rare evidence has shown that bortezomib is associated with Guillain-Barré syndrome (GBS). In the present study, a case of GBS is reported in an MM patient finishing the first course of bortezomib therapy. Based on a bone marrow cell examination revealing 34\% plasma cell infiltration and according to the International Staging System, the patient was diagnosed with stage III MM ( $\lambda$ type). Thus, a chemotherapy protocol was performed, which consisted of bortezomib and dexamethasone. However, 5 days after the first cycle, paresthesias of the upper and lower limbs developed. Standard nerve conduction studies revealed marked reduction of the motor conduction velocity of the median nerve bilaterally and of the right ulnar nerve, while sensory conduction was significantly slowed in the majority of the nerves of the upper and lower limbs. However, there was no evidence of demyelination. With this clinical evidence, a diagnosis of GBS was considered. The patient was subsequently treated with high-dose intravenous immunoglobulins (IVIGs; $400 \mathrm{mg} / \mathrm{kg} / \mathrm{day}$ for 5 days). Following IVIG treatment, the symptoms were largely relieved. This study suggested that GBS may occur when administering bortezomib, and that high-dose IVIGs could treat the symptoms of GBS.
\end{abstract}

Correspondence to: Dr Xuemei Sun, Department of Hematology, Jiangsu Province Hospital Affiliated to Nanjing University of Traditional Chinese Medicine, 155 Hanzhong Road, Nanjing, Jiangsu 210029, P.R. China

E-mail: wokibb@gmail.com

Key words: Guillain Barré syndrome, bortezomib, multiple myeloma, case report, anticancer drug

\section{Introduction}

Multiple myeloma (MM) is a hematological malignancy characterized by the accumulation of monoclonal plasma cells ( $>10 \%$ by definition) in the bone marrow (1). Chemotherapy and autologous transplantation is the major therapeutic tool for MM. It is a slowly progressing disease with a median survival of 5 years (2).

Guillain-Barré syndrome (GBS) is an autoimmune disease affecting the peripheral nervous system and is generally triggered by an acute infection. Plasma exchange or intravenous immunoglobulin, together with general supportive care has improved the outcome of GBS dramatically; the mortality in the most severe patients has fallen from $30 \%$ to $5 \%$ (3).

Bortezomib, a selective, reversible proteasome inhibitor is extensively used in patients with multiple myeloma (MM), and peripheral neuropathy (PN) is one of the most common and severe side-effects. Bortezomib-induced peripheral neuropathy (BIPN) is a small-fiber sensory neuropathy that is characterized by distal symmetric loss of all modalities in the lower limbs (4).

The current study presents the case of a patient with MM who developed Guillain-Barré syndrome (GBS) after the first course of bortezomib therapy. Written informed consent was obtained from the patient.

\section{Case report}

A 67-year-old male with an unremarkable medical history was transferred to Jiangsu Province Hospital Affiliated to Nanjing University of Traditional Chinese Medicine (Nanjing, Jiangsu, China) in November 2013 with fatigue and unintended weight loss. The laboratory examinations at admission showed the following results: Hemoglobin, $68 \mathrm{~g} / 1$ (normal ranges: $120-160 \mathrm{~g} / \mathrm{l})$; serum albumin, $36.4 \mathrm{~g} / 1$ (40-55 g/l); globulin, $29.6 \mathrm{~g} / 1(20-35 \mathrm{~g} / \mathrm{l})$ [with a low level of immunoglobulin (Ig)A, $0.17 \mathrm{~g} / 1(0.82-4.53 \mathrm{~g} / \mathrm{l}) ; \mathrm{IgG}, 5.14 \mathrm{~g} / \mathrm{l}(7.51-15.60 \mathrm{~g} / \mathrm{l})$; and $\mathrm{IgM}$, $0.08 \mathrm{~g} / \mathrm{l}(0.46-3.04 \mathrm{~g} / \mathrm{l})$ ]; urea, $11.04 \mathrm{mmol} / \mathrm{l}(2.5-7.5 \mathrm{mmol} / \mathrm{l})$; creatinine, $327.5 \mathrm{mmol} / 1(44-110 \mathrm{mmol} / \mathrm{l})$; blood free light chain $x$ (FLC $x), 329 \mathrm{mg} / 1$ (598-1329 mg/l); blood FLC $\lambda$, $1,862 \mathrm{mg} / \mathrm{l}(280-665 \mathrm{mg} / \mathrm{l})$; urine FLC $x, 3.56 \mathrm{mg} / \mathrm{l}(<5.1 \mathrm{mg} / \mathrm{l})$; urine FLC $\lambda 1,670 \mathrm{mg} / 1(<5.0 \mathrm{mg} / \mathrm{l})$; serum $\beta 2$ micro $\neg$ globulin ( $\beta 2 \mathrm{MG}), 19.31 \mu \mathrm{g} / 1(0.9-2.7 \mu \mathrm{g} / \mathrm{l})$; urea $\beta 2 \mathrm{MG}, 5.0 \mu \mathrm{g} / 1$ 
$(<0.2 \mu \mathrm{g} / \mathrm{l})$; and $24 \mathrm{~h}$ proteinuria, $9,831 \mathrm{mg} / 1(<150 \mathrm{mg} / \mathrm{l})$. The patient had normal-sized kidneys, as demonstrated by ultrasound scan. The skeletal examination was normal, and the serum calcium levels were within the normal range. A bone marrow aspiration was performed and according to the International Staging System, the patient was diagnosed with grade III MM ( $\lambda$ type) based on a bone marrow cell examination with $34 \%$ plasma cell infiltration (5). A chemotherapy protocol was followed consisting of bortezomib $\left(1.3 \mathrm{mg} / \mathrm{m}^{2}\right.$ on days 1, 4, 8 and 11, and every 21 days thereafter; Xian Janssen Pharmaceutical Ltd., Beijing, China) and dexamethasone (40 mg on days 1, 4, 8 and 11, and every 21 days thereafter; ROMIT Pharmaceutical Corporation Jiangsu, Taixing, China). However, 5 days after the first cycle, the onset of paresthesia of the upper and lower limbs occurred. Neurological examination showed that limb muscle strength and muscular tension were normal. The patient was prescribed mecobalamin tablets $(0.5 \mathrm{mg}$, 3 times a day; Eisai China Inc, Shenyang, China) for the possible diagnosis of BIPN ([National Cancer Institute-Common Toxicity Criteria grade 1) (6). However, the patient's symptoms worsened. After 1 week at home, the patient returned to the hospital. The patient's main symptom was progressive weakness and numbness of the leg and hand, ascending from the distal lower limbs to the proximal lower limb and arm muscles. The patient also presented with unsteadiness of gait. No bulbar symptoms, fasciculations, cramps, autonomic symptoms, muscle atrophy or respiratory problems were noted, and no headaches or preceding history of infection or illness were present. The patient's vital signs were stable. Neurological examination showed Medical Research Council (MRC) 4-5/5 muscle strength in the lower limbs, MRC 3-4/5 muscle strength in the upper limbs and stocking-glove sensory disturbances. The ankle jerk reflexes were lessened, while the knee jerk reflexes had disappeared bilaterally. The patient could only stand and walk on a widened base.

Although new symptoms were present, it was notable that the patient experienced a partial remission, with a decrease in the serum $\mathrm{M}$ protein and urinary $\mathrm{M}$ protein levels to $0.76 \mathrm{~g} / \mathrm{l}$ and $0.18 \mathrm{~g} / 24 \mathrm{~h}$, respectively. Serological determinations for cytomegalovirus, syphilis, Epstein-Barr virus, Brucella, human immunodeficiency virus and Borrelia were negative. Serum folate, vitamin B12 and calcium levels were within normal limits. Scans of the brain using computed tomography and gadolinium-enhanced magnetic resonance imaging did not show any abnormalities.

Nerve conduction studies (NCS) showed significant decline ( $>40 \%$ of the normal mean) in the motor conduction velocity of the median nerve bilaterally and of the right ulnar nerve, with markedly prolonged latencies of the median nerve bilaterally and of the right peroneal nerve. Sensory conduction was noticeably slower in the majority of the nerves of the upper and lower limbs.

Standard NCS were suggestive of axonal motor sensory neuropathy involving the upper and lower extremities, with no evidence of demyelination. Lumbar puncture was performed and the result demonstrated an elevated cerebrospinal fluid (CSF) protein content of $1.1 \mathrm{~g} / \mathrm{l}$. The CSF biochemistry, cytology, cell counts and culture were otherwise unremarkable.

With the clinical evidence of acute onset motor sensory quadriparesis, autonomic neuropathy and characteristic NCS findings, a diagnosis of GBS was considered. The patient was subsequently treated with high-dose IVIGs (400 mg/kg/day for 5 days). Following IVIG treatment, the patient experienced a notable recovery. At 1 week post-treatment, the only remaining symptom was mild paresthesias of the upper and lower limbs. The patient's physical examination was normal. Bortezomib was discontinued to prevent further neurotoxicity. Following the discontinuation of bortezomib treatment, the patient continued to receive dexamethasone (40 mg on days $1-4$, for 4 weeks; ROMIT Pharmaceutical Corporation) and compound cyclophosphamide (600 mg on days 1-4 for 4 weeks; Zheijiang Hisun Pharmaceutical Co., Ltd., Taizhou, China). The patient has not suffered a new PN attack for 8 months.

\section{Discussion}

Progressive, symmetrical and usually ascending weakness, and diminished or absent reflexes are the main characteristics of GBS (7). Nerve conduction velocities and amplitude are usually decreased, and CSF analysis reveals a high protein content with a normal cell count. The pattern and evolution of the neurological syndrome, the electrodiagnostic features and the CSF study in the present patient were consistent with a diagnosis of GBS. It has been shown that the pathogenesis of GBS may be an abnormal immune response to an antecedent event, most commonly an infection (8). While approximately two-thirds of patients report an illness that is respiratory or gastrointestinal nature in the weeks prior to the onset of symptoms (8), in the present patient, no clinical symptoms or signs were present to suggest an antecedent infection, and the laboratory tests, including a full blood count, serologies, urinalysis and cultures, were normal. Furthermore, several other common causes for autonomic neuropathy, in particular metabolic (diabetes and hypovitaminosis), inflammatory (autoantibodies) or toxic (alcohol), were ruled out by investigation.

Indeed, PN develops as a disease-related complication in newly diagnosed patients with MM and as a side-effect of therapy for MM (9). Direct plasma cell infiltration of the nerves was unlikely in the present patient, as there were no signs or symptoms of nerve infiltration. The clinical effect of the first chemotherapy treatment was manifested as a partial response in the patient, as evaluated by immunofixation electrophoresis.

Subsequent to excluding the other causes of GBS, the patient was diagnosed with GBS caused by bortezomib. This was a diagnosis of exclusion and should be considered when other reasonable causes of GBS are not present. The reported incidence of BIPN is $31-64 \%$, with severe (grade 3 or 4 ) symptoms observed in $3-22 \%$ of patients $(10,11)$. BIPN tends to be considered as a cumulative toxic effect, however, recent studies $(12,13)$ did not find a clear linear correlation between the cumulative dose or dose intensity and severity of polyneuropathy, indicating that certain patients developed a severe polyneuropathy following a relatively low dose of bortezomib and vice versa. The pathophysiology of bortezomib-induced neuropathy is not completely clear yet. MM could be a risk factor for the development of bortezomib-induced GBS in the present case. In conclusion, although rare, the association of bortezomib with GBS should be considered, even after the first course of treatment. 


\section{References}

1. Kyle RA and Rajkumar SV: Criteria for diagnosis, staging, risk stratification and response assessment of multiple myeloma. Leukemia 23: 3-9, 2009.

2. Morgan GJ and Davies FE: Evolving treatment strategies for myeloma. Br J Cancer 92: 217-221, 2005.

3. Winer JB: Treatment of Guillain-Barré syndrome. QJM 95: 717-721,2002.

4. Velcade ${ }^{\circledR}$ (bortezomib) [package insert]. Millennium Pharmaceuticals, Inc., Cambridge, MA, USA, 2008.

5. Greipp PR, San Miquel J, Durie BG, Crowley JJ, Barlogie B, Bladé J, Boccadoro M, Child JA, Avet-Loiseau H, Kyle RA, et al: International staging system for multiple myeloma. J Clin Oncol 23: 3412-3420, 2005

6. Brooke MH, Fenichel GM, Griggs RC, Mendell JR, Moxley R, Miller JP and Province MA: Clinical investigation in Duchenne dystrophy: 2.Determination of the "power" of therapeutic trials based on the natural history. Muscle Nerve 6: 91-103, 1983.

7. Yuki N and Hartung HP: Guillain-Barré syndrome. N Engl J Med 366: 2294-2304, 2012

8. Rosen BA: Guillain-Barré Syndrome. Pediatr Rev 33: 164-170, 2012.
9. Richardson PG, Laubach JP, Schlossman RL, Mitsiades C and Anderson K: Complications of multiple myeloma therapy, part 1 Risk reduction and management of peripheral neuropathy and asthenia. J Natl Compr Canc Netw 8 (Suppl 1): S4-S12, 2010.

10. Richardson PG, Briemberg H, Jagannath S, Wen PY, Barlogie B, Berenson J, et al: Frequency, characteristics, and reversibility of peripheral neuropathy during treatment of advanced multiple myeloma with bortezomib. J Clin Oncol 24: 3113-3120, 2006.

11. Richardson PG, Sonneveld P, Schuster MW, Irwin D, Stadtmauer EA, Facon T, et al; Assessment of Proteasome Inhibition for Extending Remissions (APEX) Investigators: Bortezomib or high-dose dexamethasone for relapsed multiple myeloma. N Engl J Med 352: 2487-2498, 2005.

12. Dimopoulos MA, Mateos MV, Richardson PG, Schlag R, Khuageva NK, Shpilberg O, et al: Risk factors for, and reversibility of, peripheral neuropathy associated with bortezomib-melphalan-prednisone in newly diagnosed patients with multiple myeloma: Subanalysis of the phase 3 VISTA study. Eur J Haematol 86: 23-31, 2011.

13. Rampen AJ, Jongen JL, van Heuvel I, Scheltens-de Boer M, Sonneveld P and van den Bent MJ: Bortezomib-induced polyneuropathy. Neth J Med 71: 128-133, 2013. 